PAPERS

\title{
Design and analysis of two degree of freedom tilt integral derivative controller with filter for frequency control and real time validation
}

\author{
Jagan Mohana Rao Chintu, Rabindra Kumar Sahu*, Sidhartha Panda ${ }^{1}$
}

\begin{abstract}
To deliver steady electric power with decent quality, intelligent and healthy control schemes are greatly necessary for automatic generation control (AGC) of power systems therefore, this work presents the design of two degree of freedom based tilt integral derivative controller with filter for AGC. Firstly, a two-area reheat thermal model is considered, and the gain of the controller are optimized by adaptive differential evolution method. The advantage of the suggested approach is validated by equating results with latest published approaches such as symbiotic organism search algorithm and articial bee colony tuned PID controller. Further, the suggested method is extended to a three unequal area thermal model and the performance of results are equated with teaching-learning based optimization-PIDD and firey algorithm-PID for the identical model. Lastly, the experiment of the proposed scheme has been employed in real-time simulation using OPAL-RT, for validation of its viability and cogency.

K e y w or ds: automatic generation control (AGC), two degree of freedom (2DOF), tilt integral derivative controller with filter (TIDF), adaptive differential evolution (ADE) algorithm, robustness analysis
\end{abstract}

\section{Introduction}

Development in size and expanding load demand is one of the key issues of interconnected power systems (PSs). This requires the work of a progressively strong enhancement method that blends the job of an increasingly vigorous beneficial control instrument for the activity of power grid. During a network of the power grid, the revision in load not exclusively influences the bus voltages but also the frequency $[1,2]$. The customary recurrence and tieline power trade should be kept up with ruffled burden load demand for the stable activity of the power grid. The strategy for automatic generation control (AGC) component utilizes a controller/advancement procedure to deal with the produced yield controls considering expanding or diminishing in load demand [3].

For the optimal execution of the power system, keeping up a recurrence to its planned worth has stayed a significant assignment in AGC. Normally, the AGC execution relies on the kind of strengthening controllers being utilized and its tuning procedures [4]. In this response, various strategies have been formed in the past for the structure of a valuable control system of AGC issues. These are the traditional controller [5], the ideal controller [6-7], the fuzzy logic controller (FLC) [8-10] and artificial neural network (ANN) [11]. Sahu et al [12] have analyzed the tilt integral derivative controller with filter (TIDF) for multi-area PSs. Kalyan et al [13] have analysed both automatic voltage regulation (AVR) and load frequency control (LFC) model using PID based differential evolution artificial electric field algorithm (DE-AEFA). In [14], authors have studied TLBO based 2-DOFPID structure for 2-area thermal system. The advantage of the suggested method has been equated the results with others. Gozde et al [15] have presented the artificial bee colony (ABC) method for AGC. The authors have shown the ability of ABC based PI, PID controllers and shown superior performance of ABC technique to PSO approach for different cost functions. Hasan, $\mathrm{N}$ et al [16] have discussed PID based hybrid taguchi-genetic algorithm (HTGA) for AGC of the system and the dynamic response of HTGA based AGC approach is evaluate to the genetic algorithm (GA) and conventional based PID controller in the interconnected model. Guha et al in [17] introduced a differential search algorithm (DSA) for the dissimilar area of thermal systems by considering PIDF controller and shown its capability by having a comparative investigation with other methods. Shabani et al in [18] discussed a PID control method optimized by the imperialist competitive algorithm (ICA) for AGC to reduce the effect of disturbance. Topno et al [19] have introduced a tilt integral derivative (TID) controller for frequency control of interconnected systems optimized by performance indexbased technique. Simhadri et al [20] has discussed the AGC problem and investigated the formulation of whale optimization algorithm (WOA) based 2DOF state feedback controller (2 DOFSFC). Khokhar et al [21] have suggested fractional order PID plus second-order derivative controller with LFC problem. Later, the effect of generation rate constraint (GRC) was introduced in these studies and the performance of DE based 2DOF-PID controller has been exhibited [22].

\footnotetext{
${ }^{1}$ Department of Electrical and Electronics Engineering, VSSUT, Burla, Sambalpur, Odisha, India, Pin-768018, * Corresponding author: rksahu_ee@vssut.ac.in
}

DOI:10.2478/jee-2020-0053, Print (till 2015) ISSN 1335-3632, On-line ISSN 1339-309X

(C) This is an open access article licensed under the Creative Commons Attribution-NonCommercial-NoDerivs License (http: //creativecommons.org/licenses/by-nc-nd/3.0/). 
Literature review illustrates that the system performance not only depends on the optimization method but also on the structure of the controller. In latest study, evolutionary optimization techniques ie . ABC [15], ICA [18], DE [23], MFO [24], SOSA [25], TLBO [26] BFOA [27] and FA [28] have been applied to for AGC problems. The studies show the way of evaluating more stable operating state for AGC problem.

There are several mechanisms concerning to frequency control of PSs by using different optimization methods and controllers. Nevertheless, there are a very trivial amount of studies correlated to intelligent controllers for frequency regulation problem. The key inspiration of current study is to improve novel methods for frequency control of PSs by offering expansions in the optimization method along with in controller configuration. In the current paper, a new controller such as 2DOF-IDF is proposed. To optimize the gain of the controller parameters ADE method is used.

The originality of the current work is presented below

- A novel 2DOF-TIDF controller is proposed for frequency control problem.

- ADE method is engaged where crossover probability rate $(\mathrm{C})$ and scaling factor $(\mathrm{F})$ are changed through the exploration process.

- The benefits of suggested ADE based 2DOF-TID controller is verified over published works.

- Stability studies in terms of phase margin and the gain margin are performed.

- Performance of the 2DOF-TID controller is authenticated considering random pulse load patterns.

- For experimental confirmation of the suggested method, results are equated with real-time simulation.

\section{System modeling}

In this article, two test models are considered for analysis of AGC. The first test system is considered as a 2-area reheat thermal model as appeared in Fig. 1 [15, 25] and the second test system is a 3 -area thermal model as appeared in Fig. 2 [26,28]. The relevant nominal values of the first test system are taken from reference $[15,25]$. The second test system is a three unequal area thermal model which is included with governor dead band (GDB) and GRC. The corresponding nominal values are taken from reference [26,28]. The suggested models are designed and analysed in SIMULINK under MATLAB. For this investigation, $3 \%$ of GRC and $0.036 \mathrm{~Hz}$ of GDB are considered $[26,28]$.

\section{Two degree of freedom - TIDF controller}

Nonlinear control structures typically carriage significantly higher document, design, application, and repairs demands than linear control structures. TIDF has benefited over the traditional controller. In a TID, the derivative approach surges the stability of the system and upsurges speed of the controller response [12]. In AGC problems, TID controllers are applied as it deals easy tuning, well disturbance rejection ratio and has fewer effects of plant parameter deviations on closed-loop response [12]. A TIDF controller is considered by substituting the proportional component of the PID by a tilted component which has transfer function (TF) of $s^{-\frac{1}{n}}$. Degree of Freedom (DOF) states that as the number of closed-loop transfer functions can be controlled separately in a control structure. A 2DOF structure normally has extra profits than a 1 DOF structure as various factors are required to be controlled for assembly the performance criteria of the control system [22]. The basic design of the suggested 2DOF-TIDF controller as appeared in Fig. 3. The $Y(s)$ is feedback signal, $U(s)$ is output signal and $R(s)$ denotes reference signal.

In this structure, control gain values $s w \& p w$ are called as derivative and proportional set points, $K_{\mathrm{P}}$ (proportional), $K_{\mathrm{I}}$ (integral), $K_{\mathrm{D}}$ (derivative) are gain values, $n$ is fractional integrator and $N$ is a derivative filter.

The controller error input and the corresponding area control errors (ACE) are as follow:

$$
\begin{gathered}
e_{1}(t)=A C E_{1}=B_{1} \Delta F_{1}+\Delta P_{\text {err }}, \\
e_{2}(t)=A C E_{2}=B_{2} \Delta F_{2}+a_{12} \Delta P_{\text {err }} .
\end{gathered}
$$

The ADE algorithm is applied to tuning the optimal gain of the 2DOF-TIDF controller with the objective function ITAE (integral of time multiplied absolute error) to be minimized. The formulation for ITAE is

$$
J=\int_{0}^{t_{\mathrm{S}}}\left(\left|\Delta F_{j}\right|+\left|\Delta P_{\mathrm{jk}}\right|\right) t \mathrm{~d} t .
$$

In the expression $\Delta F_{j}$ is frequency deviation in area $j$; $\Delta P_{j k}$ is the change in tie-line power between area $j$ and $k ; t_{\mathrm{S}}$ is the simulation time.

The optimization problem is defined as Minimize $J$ Subject to

$$
\begin{aligned}
& n_{\min } \leq n \leq n_{\max } \\
& K_{P_{\min }} \leq K_{P} \leq K_{P_{\max }} \\
& p w_{\min } \leq p w \leq p w_{\max } \\
& K_{I_{\min }} \leq K_{I} \leq K_{I_{\max }} \\
& s w_{\min } \leq s w \leq s w_{\max } \\
& N_{\min } \leq N \leq N_{\max } \\
& K_{D \min } \leq K_{D} \leq K_{D \max }
\end{aligned}
$$

Here $J$ refers to the objective function. 


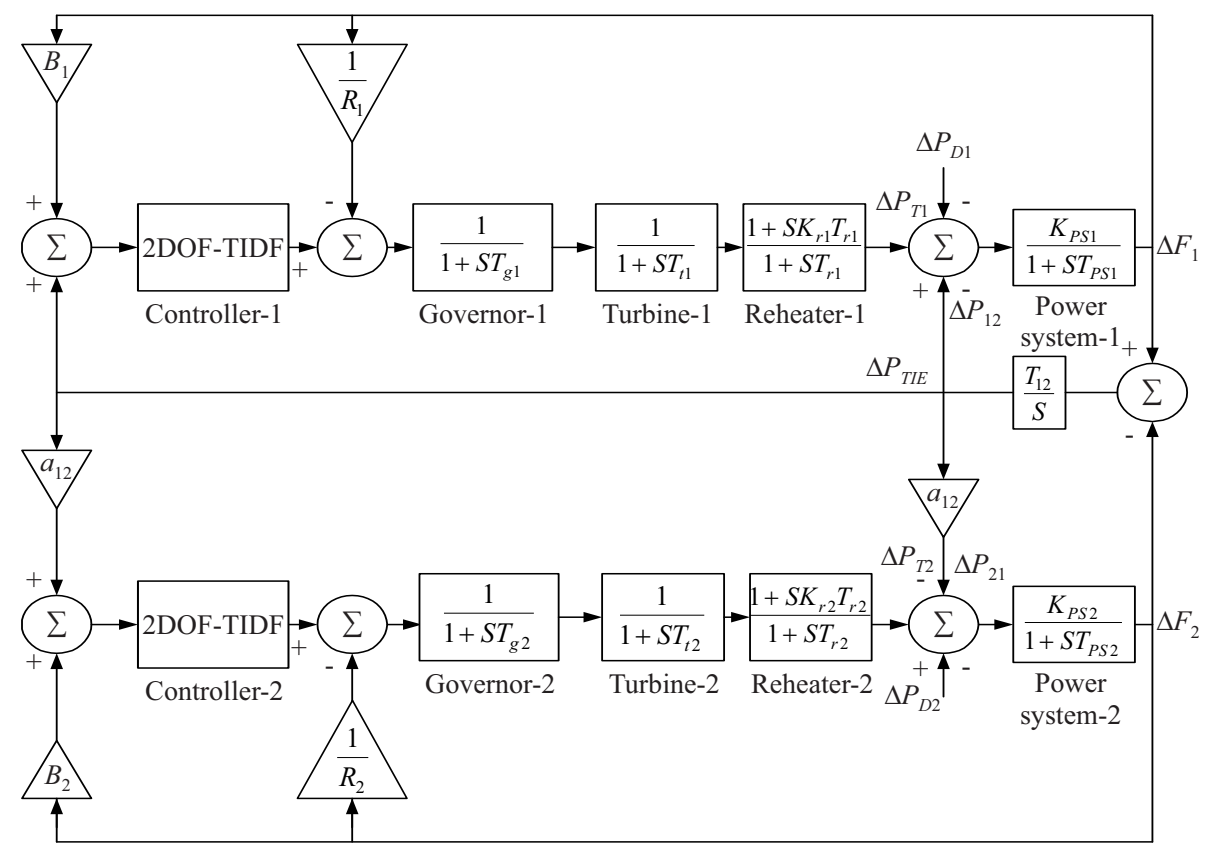

Fig. 1. 2-Area system

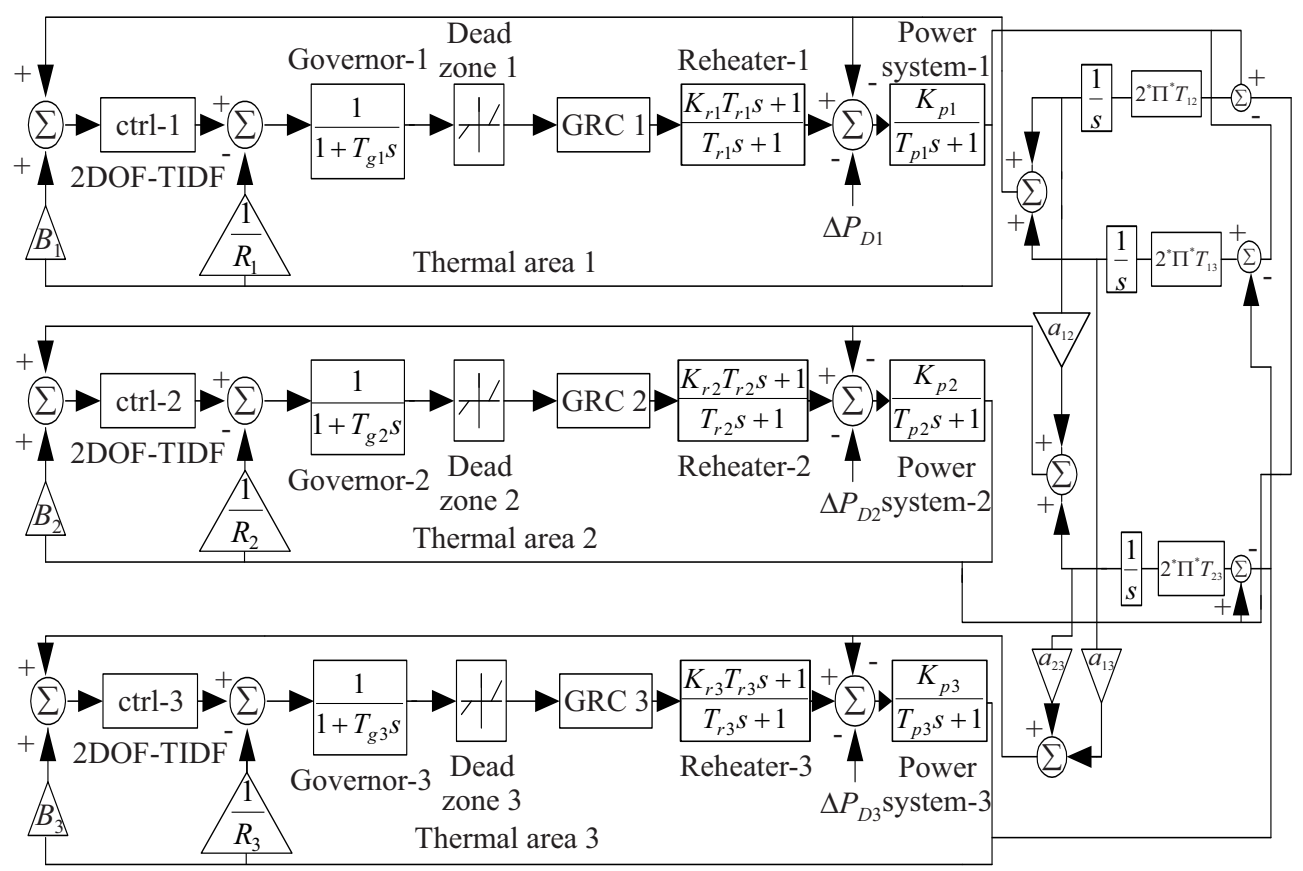

Fig. 2. 3-Area system

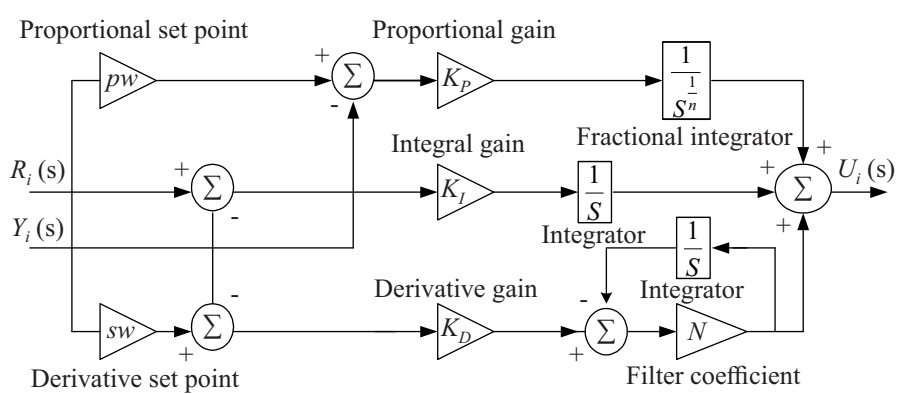

Fig. 3. Design of DOF-TIDF controller 
Table 1. Parameters of 2DOF-TIDF \& TIDF controllers

\begin{tabular}{|c|c|c|c|c|c|}
\hline \multirow{2}{*}{\multicolumn{2}{|c|}{$\begin{array}{l}\text { Controller } \\
\text { parameters }\end{array}$}} & \multicolumn{2}{|c|}{ 2-area system } & \multicolumn{2}{|c|}{3 -area system } \\
\hline & & TIDF & $\begin{array}{l}2 \mathrm{DOF} \\
-\mathrm{TIDF}\end{array}$ & TIDF & $\begin{array}{l}2 \mathrm{DOF} \\
-\mathrm{TIDF}\end{array}$ \\
\hline \multirow{7}{*}{ Area-1 } & $p w$ & - & 0.2306 & - & 0.3136 \\
\hline & $s w$ & - & 0.5202 & - & 1.6052 \\
\hline & $K_{\mathrm{P}}$ & -4.8809 & 9.9056 & 0.1696 & 0.5794 \\
\hline & $K_{\mathrm{I}}$ & -4.6378 & 6.7500 & -0.4402 & 0.6726 \\
\hline & $K_{\mathrm{D}}$ & -1.4716 & 5.0014 & -0.1157 & 0.3908 \\
\hline & $n$ & 8.4291 & 8.1164 & 8.9825 & 1.1598 \\
\hline & $N$ & 217.797 & 487.851 & 177.416 & 8.847 \\
\hline \multirow{7}{*}{ Area-2 } & $p w$ & - & 1.7672 & - & 0.4957 \\
\hline & $s w$ & - & 1.2818 & - & 1.2629 \\
\hline & $K_{\mathrm{P}}$ & -3.5581 & 8.0917 & -0.3522 & 0.0218 \\
\hline & $K_{\mathrm{I}}$ & -1.8806 & 6.2144 & -0.0145 & 0.3691 \\
\hline & $K_{\mathrm{D}}$ & -3.7349 & 2.1208 & -0.0856 & 0.1768 \\
\hline & $N$ & 8.7176 & 8.8588 & 4.1498 & 8.8145 \\
\hline & $N$ & 223.098 & 78.449 & 239.373 & 178.411 \\
\hline \multirow{7}{*}{ Area-3 } & $p w$ & - & - & - & 0.0141 \\
\hline & $s w$ & - & - & - & 1.8034 \\
\hline & $K_{\mathrm{P}}$ & - & - & -0.0528 & 0.4568 \\
\hline & $K_{\mathrm{I}}$ & - & - & -0.0839 & 0.9777 \\
\hline & $K_{\mathrm{D}}$ & - & - & -0.8815 & 0.4684 \\
\hline & $n$ & - & - & 9.8549 & 3.7718 \\
\hline & $N$ & - & - & 21.266 & 183.609 \\
\hline
\end{tabular}

\section{Adaptive DE optimization technique}

Inspired by the evolution strategy, DE algorithms are found to be an efficient problem-solving approach for complex engineering problems [29]. It has three stages of operation.

\subsection{Mutation}

The mutant vector is to be initiated for every parent vector, on every generation i.

$$
\left.w^{j}\right|_{i}=\left.y^{q 1}\right|_{i}+F\left(\left.y^{q 2}\right|_{i}-\left.y^{q 3}\right|_{i}\right) .
$$

\subsection{Crossover}

It is an important mechanism for improvement in population diversity. In Binary crossover, the trial vector can be generated through the target vector and its mutant vector

$$
\left.v_{l}^{j}\right|_{i}= \begin{cases}\left.w_{l}^{j}\right|_{i} & \text { if } \operatorname{rand}(0,1) \leq C \\ \left.y_{l}^{j}\right|_{i} & \text { otherwise }\end{cases}
$$

Where and $C$ indicates the rate of crossover probability. For every vector and, the trial vector can be generated as

$$
\left.v^{j}\right|_{i}=\left.y^{j}\right|_{i}+F_{j}\left(\left.w^{j}\right|_{i}-\left.y^{j}\right|_{i}\right) \text {. }
$$

\subsection{Selection}

For a fix population size during every generation, the selection operation is used. Having the best fitness value either in the target vector or trial vector, one is to be replaced by others with the highest fitness value. The selection is represented as

$$
\left.y^{j}\right|_{i+1}= \begin{cases}\left.v^{j}\right|_{i} & \text { if } f\left(\left.v^{j}\right|_{i}\right) \leq f\left(\left.y^{j}\right|_{i}\right), \\ \left.y^{j}\right|_{i} & \text { otherwise. }\end{cases}
$$

DE is an efficient optimization method which depends on algorithmic dependent parameters. Proper selections of these values are important in context to estimate the performance of the algorithm. Its parameters such as scaling factor $(F)$ and crossover probability rate $(C)$ need to be set with proper constraint values. Many researchers have suggested some ways to select the values of these two parameters [10].

Selecting proper controller parameters is always a depending activity for a problem. Due to several iterative cycles choosing the controller parameters in a trial and error bases is timing consuming. The proposed method consisting of adaptive technique to modify the controller parameters during the simulation for generation $i$, the triangular factors such as $\Lambda F, \Lambda C$ has been used for evaluating $\left.f_{\mathrm{F}}(y)\right|_{i}$ and $\left.f_{\mathrm{C}}(y)\right|_{i}$ for every child in the population. For the proposed work Triangular Distribution factor of $F(\wedge F)$ and $C(\wedge C)$ has been used, where $\wedge F=[0.1,1,2]$ and $\wedge C=[0.1,0.4,1]$.

To choose $F^{i}$ and $C^{i}$ three values such as $m x$ (maximum), $m n$ (minimum) and $m d$ (median) are used in a set in the generation and are

$$
\begin{gathered}
F^{i}=\left\{\begin{array}{c}
F_{m n}+\sqrt{V_{\mathrm{rnd}}\left(F_{m x}-F_{m n}\right)\left(F_{m d}-F_{m n}\right)} \\
\text { if } V_{\mathrm{rnd}}<\left(F_{m x}-F_{m n}\right)\left(F_{m d}-F_{m n}\right), \\
F_{m x}-\sqrt{\left(1-V_{\mathrm{rnd}}\right)\left(F_{m x}-F_{m n}\right)\left(F_{m x}-F_{m d}\right)} \\
\text { otherwise. }
\end{array}\right. \\
C^{i}=\left\{\begin{array}{c}
C_{m n}+\sqrt{V_{\mathrm{rnd}}\left(C_{m x}-C_{m n}\right)\left(C_{m d}-C_{m n}\right)} \\
\text { if } V_{\mathrm{rnd}}<\left(C_{m x}-C_{m n}\right)\left(C_{m d}-C_{m n}\right) \\
C_{m x}-\sqrt{\left(1-V_{\mathrm{rnd}}\right)\left(C_{m x}-C_{m n}\right)\left(C_{m x}-C_{m d}\right)} \\
\text { otherwise. }
\end{array}\right.
\end{gathered}
$$

In the above equations, the value of $V_{\text {rnd }}$ has been chosen in a uniform distribution with a range of $[0,1]$.

Before executing the mutation operation $F^{i}$ and $C^{i}$ values have been chosen. Which effects mutation, cross over and selection operation of the newly generator vector $\left.y^{j}\right|_{i+1}$ rather the method of adaptive DE Seems to be complex, till it is worthy to note that values for $m x$ (maximum), $m n$ (minimum) and $m d$ (median) are used for computing adaptive $F^{i}$ and $C^{i}$ parameters during the simulation. Hence it leads to the fact for non-adjustment of additional parameters. In this proposed method due to the proper selection of the parameter $F$ and $C$, it is not required to speculate the value. Due to the simple 


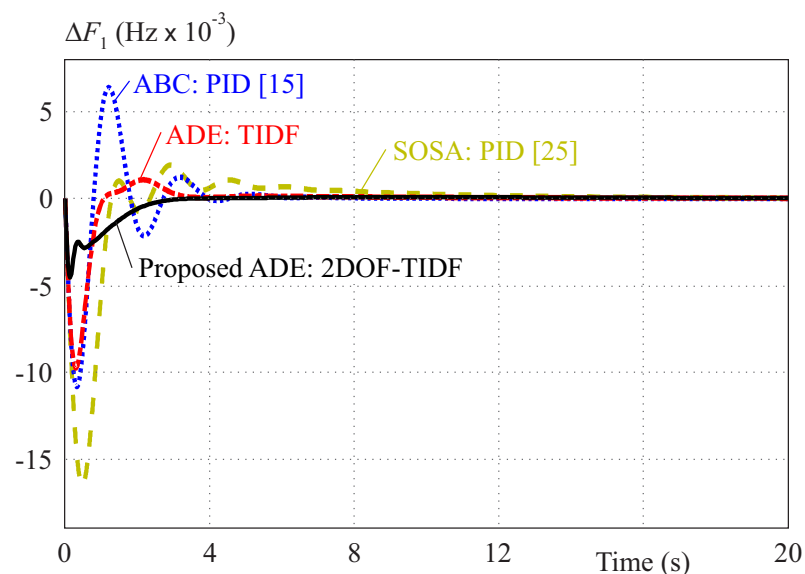

Fig. 4. Deviation in frequency of area-1 (2-area system)

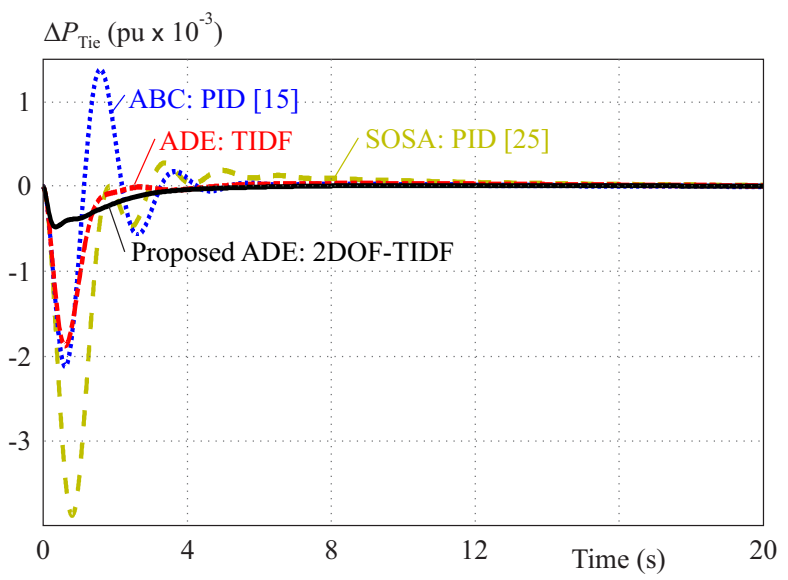

Fig. 6. Deviation in tie-line power (2-area system)

generation of adaptive values for $F$ and $C$, the proposed approach is useful to maintain steady time complexity as compared to traditional DE method.

\section{Results and discussions}

\subsection{Test system-1}

To study the frequency of both areas at the same time as tie-line power, TIDF and 2DOF-TIDF controllers are achieved independently and investigated the system in each area of the reheat thermal model. The design of the reheat thermal model is represented in Fig. $1[15,25]$. The simulation is performed with a $1 \%$ step load perturbation (SLP) in area-1 $\left(\Delta P_{\mathrm{D} 1}=0.1 \mathrm{pu} \mathrm{MW}\right)$ at $t=0$. The optimization methods were repeated 20 times. The best result is selected as the controller parameter. The optimized gain values of both controllers are listed in Tab. 1. The graphical demonstration of responses $\left(\Delta F_{1}\right.$, $\Delta F_{2}$ and $\Delta P$ ) of ABC [15]/SOSA [25] based PID and ADE based TIDF are shown in Figs. 4-6. The response indices related to undershoot $\left(U_{\mathrm{s}}\right)$, objective function and settling time $\left(T_{\mathrm{s}}\right)$ are indicated in Tab. 2. It is inspected from Tab. 2 and Figs. 4-6 that the suggested controller illustrates better dynamic performance as compared to

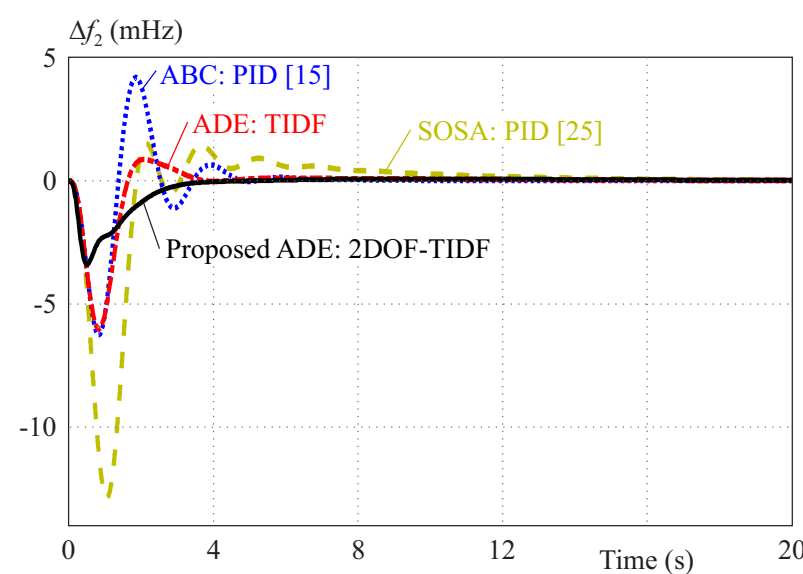

Fig. 5. Deviation in frequency of area-2 (2-area system)

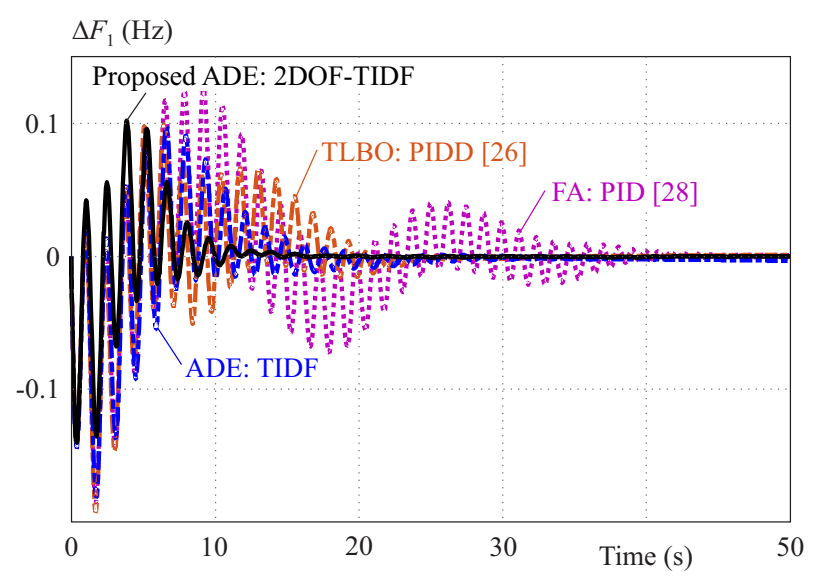

Fig. 7. Deviation in frequency of areaarea system

other recently published techniques. From Tab. 2 it can be seen that the enhancements in the objective function (ITAE) values with ADE based TIDF and 2DOF-TIDF controllers are achieved $44.58 \%$ and $47.55 \%$ respectively when compared with best claimed published result ABC based PID controller [15]. For the settling times the enhancements are: $26.16 \%$ and $52.87 \%$ for $\Delta F_{1} ; 31.44 \%$ and $45.42 \%$ for $\Delta F_{2} ; 13.51 \%$ and $29.05 \%$ for $\Delta P$ respectively with ADE tuned TIDF and 2DOF-TIDF controllers. It is clear from Table 2 is that the suggested ADE based TIDF outperforms the ABC based PID controller and best performance is achieved with ADE based 2DOF-TIDF controller.

\subsection{Test system-2}

To exhibit the effectiveness and prospective of the suggested method, it is further extended to a 3-area thermal model including GDB and GRC is represented in Fig. $2[26,28]$. The area ratings of 1 to 3 are $2000 \mathrm{MW}$, $4000 \mathrm{MW}$ and $8000 \mathrm{MW}$ respectively. A $10 \%$ SLP is used in area- 1 at $t=0$ and the tuning values of proposed ADE based 2DOF-TIDF and TIDF controller are listed in Tab. 1. The performance index values like ITAE and $T_{\mathrm{S}}$ are indicated in Tab. 3. The enhancements are $11.16 \%$ and $62.98 \%$ in the ITAE with ADE based TIDF and 


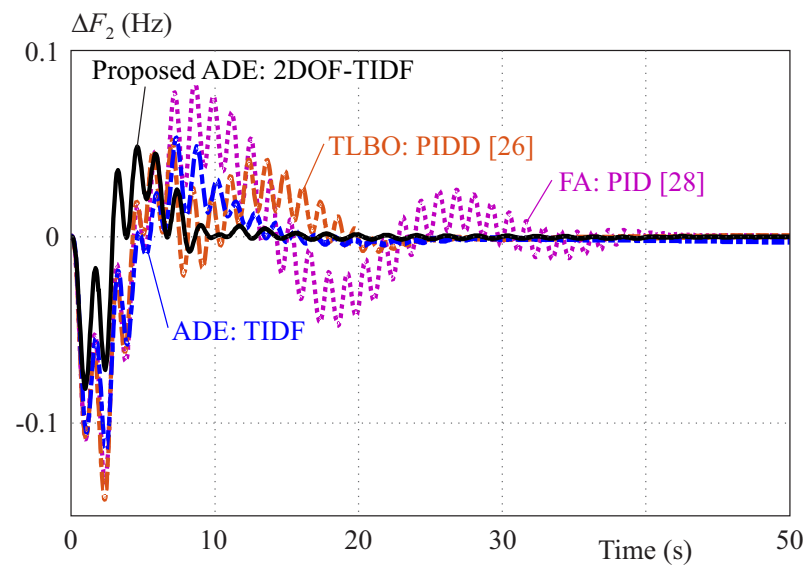

Fig. 8. Deviation in frequency of area-2 (3-area system

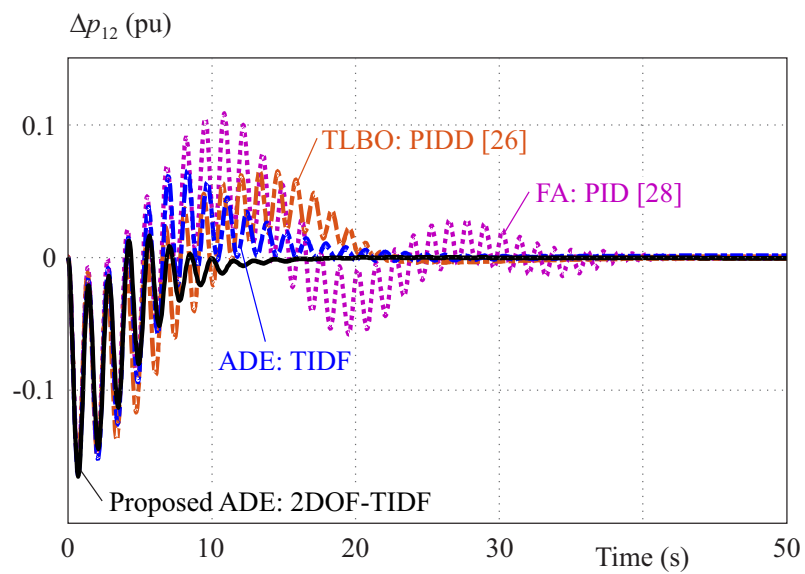

Fig. 10. Deviation in tie-line power between area $1 \& 2$

Table 2. Performance index values (2-area system)

\begin{tabular}{|c|c|c|c|c|c|c|}
\hline \multicolumn{2}{|c|}{ Parameters } & $\begin{array}{c}\text { ADE: } \\
\text { 2DOF- } \\
\text { TIDF }\end{array}$ & $\begin{array}{l}\text { ADE } \\
\text { TIDF }\end{array}$ & $\begin{array}{c}\text { ABC: } \\
\text { PID } \\
{[15]}\end{array}$ & $\begin{array}{c}\text { SOSA: } \\
\text { PID } \\
{[25]}\end{array}$ & $\begin{array}{l}\text { FA: } \\
\text { PID } \\
{[24]} \\
\end{array}$ \\
\hline \multicolumn{2}{|c|}{ ITAE $\times 10^{-3}$} & 30 & 31.7 & 57.2 & 99.6 & 101.2 \\
\hline \multirow{3}{*}{$T_{\mathrm{S}}(\mathrm{s})$} & $\overline{\Delta F_{1}}$ & 2.54 & 3.98 & 5.39 & 6.60 & 9.82 \\
\hline & $\Delta F_{2}$ & 3.28 & 4.12 & 6.01 & 7.08 & 6.67 \\
\hline & $\Delta P_{\text {tie }}$ & 1.05 & 1.28 & -1.48 & -1.61 & -1.78 \\
\hline \multirow{3}{*}{$U_{\mathrm{S}} \times 10^{-3}$} & $\Delta F_{1}$ & -4.6 & -4.9 & -5.1 & -16.5 & -15.4 \\
\hline & $\Delta F_{2}$ & -2.8 & -3.1 & -3.2 & -12.8 & -11.7 \\
\hline & $\Delta P_{\text {tie }}$ & -0.5 & -0.9 & -1.0 & -3.8 & -3.5 \\
\hline
\end{tabular}

2DOF-TIDF controllers respectively when compared to best claim results TLBO based PIDD controller [26]. Such enhancements are also observed for the $T_{\mathrm{S}}$ as $1.24 \%$ and $24.58 \%$ for $\Delta F_{1} ; 0.72 \%$ and $12.40 \%$ for $\Delta F_{2} ; 0.93 \%$ and $3.98 \%$ for $\Delta F_{3}$ respectively with ADE tuned TIDF and 2DOF-TIDF controllers. From Tab. 3, it is evident that the suggested ADE based TIDF controller outperforms the TLBO based PIDD [26] \& FA based PID [28] controller. The best performance is achieved with the proposed ADE based 2DOF-TIDF controller. The com-

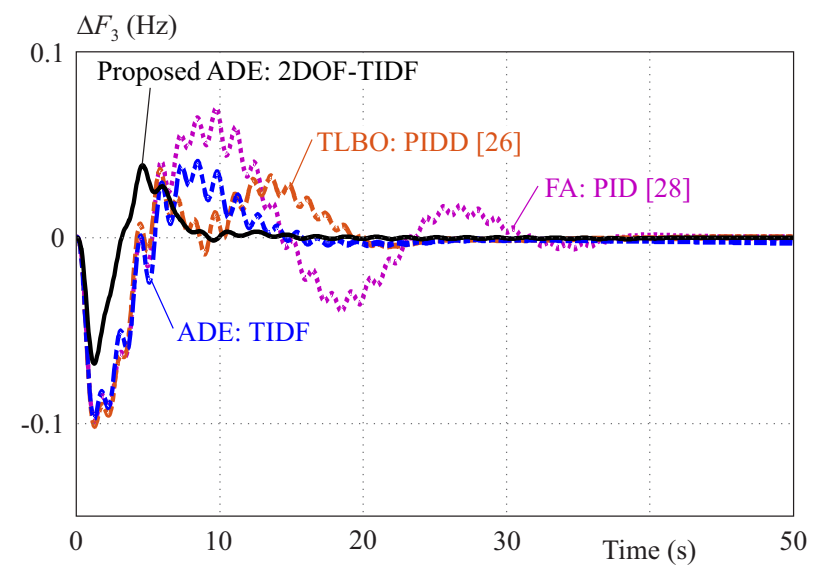

Fig. 9. Deviation in frequency of area-3 (3-area system

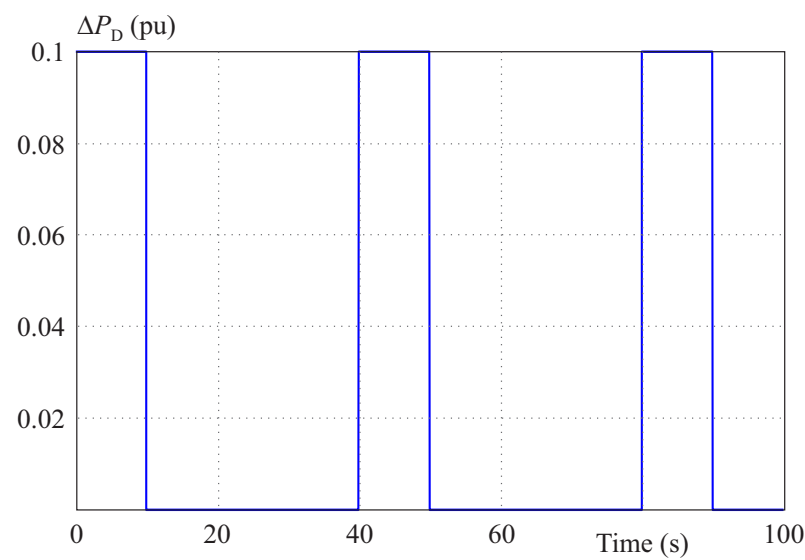

Fig. 11. Pulse load pattern

Table 3. Performance index values (3-area system)

\begin{tabular}{|c|c|c|c|c|c|}
\hline \multicolumn{2}{|c|}{ Parameters } & $\begin{array}{c}\text { ADE: } \\
\text { 2DOF- } \\
\text { TIDF }\end{array}$ & $\begin{array}{l}\text { ADE: } \\
\text { TIDF }\end{array}$ & $\begin{array}{c}\text { TLBO: } \\
\text { PIDD } \\
{[26]}\end{array}$ & $\begin{array}{l}\text { FA: } \\
\text { PID } \\
{[28]}\end{array}$ \\
\hline & TAE & 7.55 & 18.12 & 20.44 & 30.90 \\
\hline \multirow{6}{*}{$T_{\mathrm{S}}(\mathrm{s})$} & $\overline{\Delta F_{1}}$ & 14.54 & 19.04 & 19.28 & 26.56 \\
\hline & $\Delta F_{2}$ & 15.82 & 17.96 & 18.06 & 26.72 \\
\hline & $\Delta F_{3}$ & 17.58 & 18.14 & 18.31 & 26.55 \\
\hline & $\Delta P_{\mathrm{tie} 12}$ & 15.99 & 22.06 & 23.39 & 24.03 \\
\hline & $\Delta P_{\text {tie13 }}$ & 16.45 & 19.18 & 19.23 & 19.66 \\
\hline & $\Delta P_{\mathrm{tie} 23}$ & 13.95 & 15.26 & 15.84 & 20.70 \\
\hline
\end{tabular}

parative dynamic performances of the proposed approach are represented in Figs. 7-10. Further it can be observed that the suggested ADE based 2DOF-TIDF the system response is much better than that of the published approach like FA tuned PID [28], TLBO tuned PIDD [26] and ADE tuned TIDF controller. Moreover, with the suggested approach, the system response is faster and quickly suppresses the oscillations in tie-line powers and frequencies in each area. 
Table 4. Performance analysis with different circumstances (3-area system)

\begin{tabular}{ccccccccc}
\hline \multirow{2}{*}{ Parameter } & Change & \multicolumn{7}{c}{ Settling time (s) } \\
& $\%$ & $\Delta F_{1}$ & $\Delta F_{2}$ & $\Delta F_{3}$ & $\Delta P_{1} 2$ & $\Delta P_{13}$ & $\Delta P_{23}$ & ITAE \\
\hline Nominal & 0 & 14.54 & 15.82 & 17.58 & 15.99 & 16.45 & 13.95 & 7.55 \\
\hline Loading & +25 & 14.36 & 14.96 & 18.26 & 16.38 & 17.68 & 12.42 & 8.54 \\
condition & -25 & 16.55 & 17.97 & 17.10 & 19.74 & 16.09 & 12.38 & 6.73 \\
\hline \multirow{2}{*}{$T_{\mathrm{g}}$} & +25 & 18.41 & 20.46 & 18.93 & 18.28 & 16.44 & 14.22 & 7.12 \\
& -25 & 16.75 & 18.51 & 18.83 & 20.48 & 16.82 & 14.32 & 7.27 \\
\hline \multirow{2}{*}{$T_{\mathrm{t}}$} & +25 & 19.39 & 19.41 & 17.94 & 19.18 & 18.52 & 16.14 & 7.19 \\
& -25 & 15.95 & 16.52 & 17.84 & 20.69 & 16.93 & 14.35 & 7.23 \\
\hline \multirow{2}{*}{$R$} & +25 & 17.42 & 18.93 & 16.36 & 20.27 & 16.62 & 14.26 & 6.93 \\
& -25 & 15.62 & 17.99 & 18.41 & 20.46 & 18.43 & 16.64 & 8.14 \\
\hline \multirow{2}{*}{$R$} & & & & & & & &
\end{tabular}

Table 5. Stability margins for parameter variation

\begin{tabular}{cccc}
\hline Parameter & $\begin{array}{c}\text { Change } \\
(\%)\end{array}$ & $\begin{array}{c}\text { PM } \\
(\mathrm{deg})\end{array}$ & $\begin{array}{c}\text { GM } \\
(\mathrm{dB})\end{array}$ \\
\hline Nominal & 0 & 128 & 22.5 \\
\hline Loading & +50 & 152 & 26.2 \\
condition & -50 & 68.4 & 15.8 \\
\hline \multirow{2}{*}{$T_{\mathrm{g}}$} & +50 & 119 & 19.4 \\
& -50 & 138 & 28 \\
\hline \multirow{2}{*}{$T_{\mathrm{t}}$} & +50 & 98.6 & 21.9 \\
& -50 & 153 & 23.9 \\
\hline \multirow{2}{*}{$R$} & +50 & 129 & 22.2 \\
& -50 & 85.6 & 22.8 \\
\hline
\end{tabular}

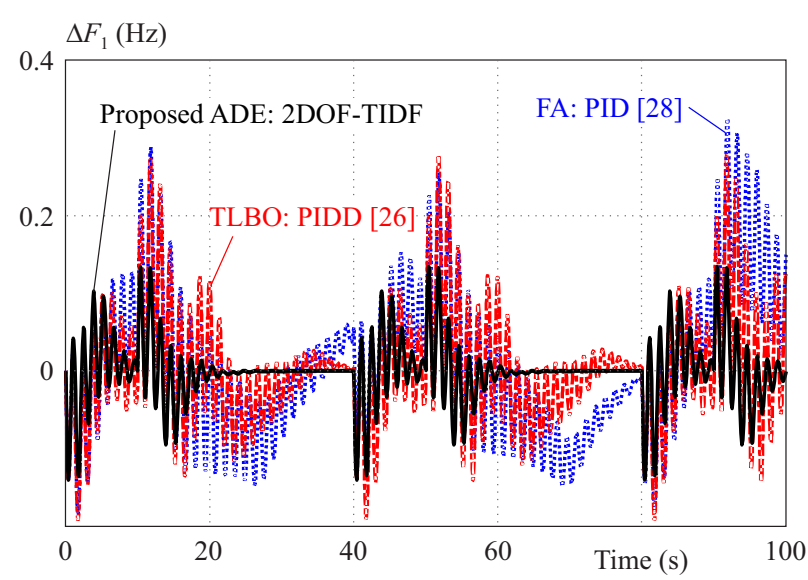

Fig. 12. $\Delta F_{1}$ under pulse load pattern

\section{Robustness and stability analysis}

The key objective of the AGC approach in the power system is to preserve the sensitivity and stability against the deviation in system parameters from their nominal values $[15,25,26,28]$. The range of the system parameters is considered $\pm 25 \%$ for second test systems. It is clear from Table 4 that the performance index values are more or less the same as with the proposed method (ADE: $2 \mathrm{DOF}-\mathrm{TIDF})$ and the effect of the deviation in system parameters on the performance is insignificant. Therefore, it can be determined that the suggested control plan offers a robust control.

To examine the efficacy of the suggested approach under pulse load pattern, pulse load changes are tested in area-1 and shown in Fig. 11. As an example, the response in frequency at area- 1 is displayed in Fig. 12. For assessment, the result of the suggested approach (ADE: 2DOF-TIDF) is equated with FA: PID [28] and TLBO:PIDD [26] controller. From Fig. 12, it can be comprehended that the suggested approach is robust and achieve acceptably under pulse load disturbances. It is moreover apparent from Fig. 12 that superior response is attained with the proposed approach to others.

For stability investigation, the laplace transform of the test systems is firstly acquired using MATLAB/Simulink model. For the ease of explanation, for the systems, simply area- 1 is considered to find the transfer function (TF) depiction. The structure parameters have been preferred with $\pm 50 \%$ range of variations. For establishing the TF along with the suggestion input at the same time as an output signal, the external disturbance \& tie-line power values are set as zero with the appropriate choice of linear analysis point and using its tool from MATLAB/Simulink [10].

The bode plot has been achieved. For the test model, results of gain margin (GM) and phase margin (PM) are listed in Tab. 5. As described in Tab. 5, the GM and PM values are positive which demonstrates the stability of the suggested controller on the existence of $\pm 50 \%$ parameter variations. As an example, the bode plot to scrutinize the deviations $T_{g}$ for the test model is indicated in Fig. 13 . It is apparent from Fig. 13 that there is no variation in frequency responses with recognition to pupation in system parameters, which settles the robustness of the control system.

\section{Experimental verification}

The Simulink model in real-time with the OPAL-RT simulator atmosphere has been assembled. A physical 

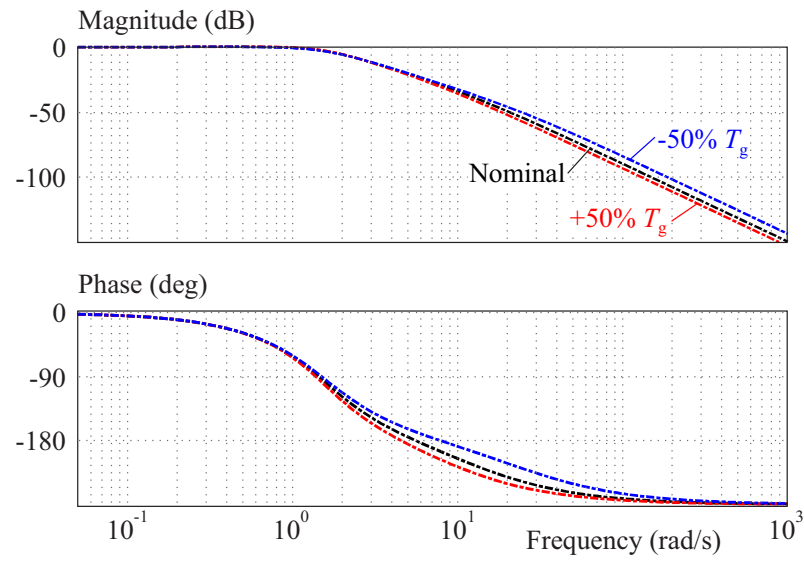

Fig. 13. Bode plot responses in $T_{g}$ variations
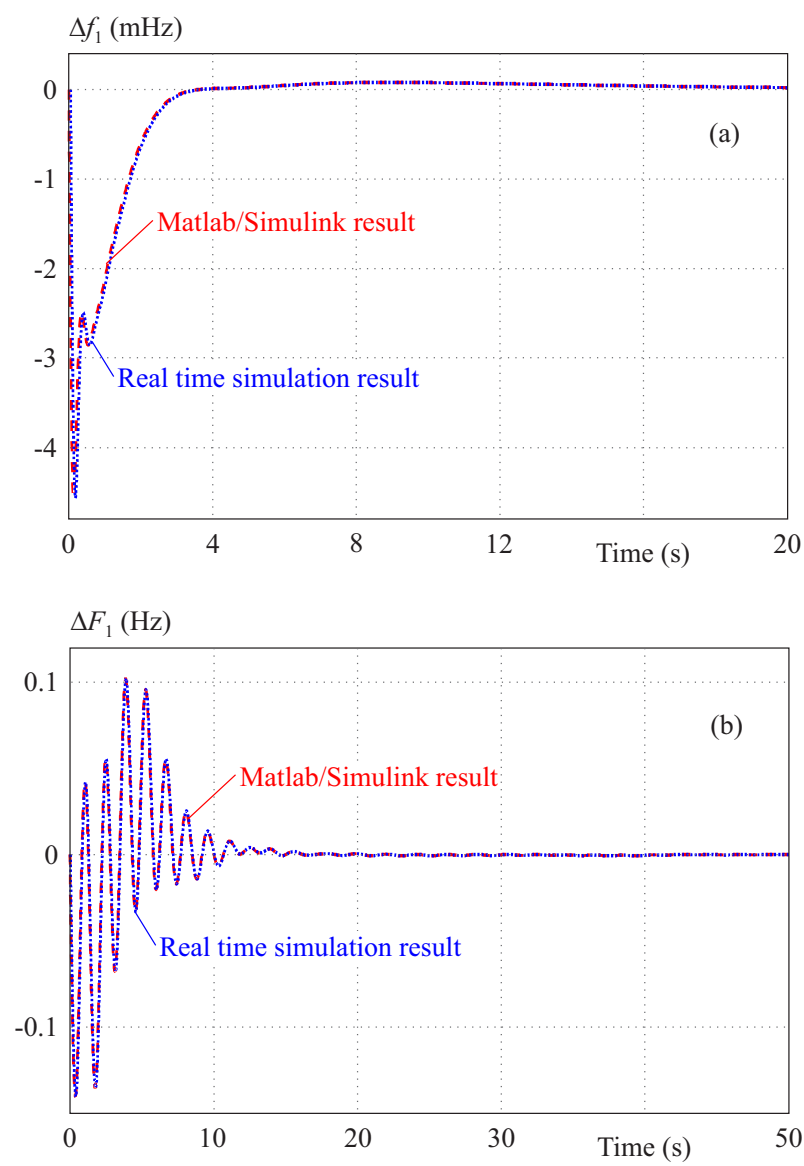

Fig. 14. Comparison of matlabsimulink and realtime simulation outcomes: (a) - 2-area system (b) - 3-area system

plant is substituted by a virtual plant on a real-time simulator (RTS). That is the actual controller is tested by a simulated model employed in the RTS. Hardware in Loop (HiL) method is harder to apply for electrical arrangements as it is obstinate by nature, and it needs very fewer time stage to achieve correctness. OPAL-RT permits premature examination of controller when physical test forms are not accessible. This has the determination to experimentally authorize the suggested control method as in off-line simulation errors and delays are inattentive. The HiL arrangement comprises a com- puter with a windows operating system as a command station. A simulation atmosphere which is applied to run the test model is established on this PC. It also comprises a router to assign all the arrangement strategies. Fixed step time is selected for the power system to run in real-time. The results of MATLAB/Simulink and RTS are shown in Figs. 14(a)-(b) for both the test systems. From Fig. 14(a)-(b), it is evident that RTS results are similar to the MATLAB/Simulink results for both test systems.

\section{Conclusions}

In the current study, an effort has been made to ADE based 2DOF-TIDF for AGC studies. Firstly, a 2-area reheat thermal model is examined. The performance of the suggested approach has been compared with some recently approach in the literature like ABC/SOSA based PID and ADE based TIDF controller for the identical test system. It is seen that the $J$ value is reduced by $47.55 \%$ with suggested ADE-2 DOFTID compared to ABC-PID [15] controller. After that, the investigation is continued to three unequal thermal models including with nonlinearities like GDB and GRC. Simulation results reveal that the dynamic performance of the proposed approach has been superior in terms of the objective function and settling time over published TLBO tuned PIDD [26] and FA tuned PID [28] controller. It is also perceived that the $J$ values are reduced by $62.91 \%$ with suggested ADE-2 DOF-TID controller while compared with published TLBO tuned PIDD [26] approach. Besides, a sensitivity analysis is executed under a $\pm 25 \%$ parameter variation for both test systems. Moreover, the simulation study conducted on the proposed approach with a pulse load pattern. The results indicate that the proposed approach method stabilizes oscillations compared to other approaches. Further stability investigation has been executed in terms of GM and PM which reveal the stability of the proposed control approach in the attendance of $\pm 50 \%$ parameter change for the test system. It is realized that the recommended ADE tuned 2DOF-TIDF controller is provides superior performance contrast to other methods. Finally, the proposed method is authenticated and executed in the HiL with OPAL-RT. The results confirm the ability of the suggested method as the RTS results and MATLAB results are same with almost no variation. Future study prospects are the presence of random renewable energy bases in the test system and application of a new optimization method for the controller scheme.

\section{REFERENCES}

[1] O. I. Elgerd, Electric Energy Systems Theory: An Introduction, 2nd ed. New Delhi: Tata McGraw-Hill, 2007.

[2] H. Bevrani, and T. Hiyama, Intelligent Automatic Generation Control, CRC press, 2011. 
[3] W. Tan, "Unified Tuning of PID Load Frequency Controller for Power Systems via IMC", IEEE Transactions on Power Systems, vol. 25 , pp. 341-50, 2009.

[4] R. K. Sahu, S. Panda, S. Padhan, "A Hybrid Firefly Algorithm and Pattern Search Technique for Automatic Generation Control of Multi Area Power Systems", International Journal of Electrical Power \& Energy Systems, vol. 64, pp. 9-23, 2015.

[5] L. C. Saikia, J. Nanda, S. Mishra, "Performance Comparison of Several Classical Controllers in AGC for Multi-Area Interconnected Thermal System", International Journal of Electrical Power \& Energy Systems, vol. 33, pp. 394-401, 2011.

[6] K. S. Parmar, S. Majhi, D. P. Kothari, "Load Frequency Control of a Realistic Power System with Multi-Source Power Generation", International Journal of Electrical Power \& Energy Systems, vol. 42, pp. 426-433, 2012.

[7] Y. Arya, and N. Kumar, "Optimal Control Strategy-Based AGC of Electrical Power Systems: A Comparative Performance Analysis", Optimal Control Applications and Methods, vol. 38, pp. 982-992, 2017.

[8] E. Çam, and I. Kocaarslan, "Load Frequency Control in Two Area Power Systems using Fuzzy Logic Controller", Energy Conversion and Management, vol. 46, pp. 233-243, 2005.

[9] Y. Arya, "Automatic Generation Control of Two-Area Electrical Power Systems via Optimal Fuzzy Classical Controller", Journal of the Franklin Institute, vol. 355, pp. 2662-88, 2018.

[10] JM. Chintu, R. K. Sahu, and S. Panda, "Adaptive Differential Evolution Tuned Hybrid Fuzzy PD-PI Controller for Automatic Generation Control of Power Systems", International Journal of Ambient Energy, pp. 1-16, 2019.

[11] S. R. Khuntia, and S. Panda, "Simulation Study for Automatic Generation Control of a Multi-Area Power System by ANFIS Approach", Applied Soft Computing, vol. 12, pp. 333-41, 2012.

[12] R. K. Sahu, S. Panda, A. Biswal, and TS. Gorripotu, "Design and Analysis of Tilt Integral Derivative Controller with Filter for Load Frequency Control of Multi-Area Interconnected Power Systems", ISA Transactions, vol. 61, pp. 251-264, 2016.

[13] C. N. S. Kalyan, and G. S. Rao, "Frequency and Voltage Stabilisation in Combined Load Frequency Control and Automatic Voltage Regulation of Multi Area System with Hybrid Generation Utilities by AC/DC Links", International Journal of Sustainable Energy, 2020, https://doi. org/10. 1080/14786451. 2020. 1797740.

[14] R. K. Sahu, S. Panda, U. K. Rout, and D. K. Sahoo, "Teaching Learning Based Optimization Algorithm for Automatic Generation Control of Power System using 2-DOF PID Controller", International Journal of Electrical Power \& Energy Systems, vol. 77, pp. 287-301, 2016.

[15] H. Gozde, MC. Taplamacioglu, and I. Kocaarslan, "Comparative Performance Analysis of Artificial Bee Colony Algorithm in Automatic Generation Control for Interconnected Reheat Thermal Power System", International Journal of Electrical Power \& Energy Systems, vol. 42, pp. 167-78, 2012.

[16] N. Hasan, I. Nasirudin, S. Farooq, "Hybrid Taguchi Genetic Algorithm-Based AGC Controller for Multisource Interconnected Power System", Electric Power Components and Systems, vol. 47, pp. 101-12, 2019.

[17] D. Guha, P. K. Roy, and S. Banerjee, "Study of Differential Search Algorithm Based Automatic Generation Control of an Interconnected Thermal-Thermal System with Governor Dead-Band", Applied Soft Computing, vol. 52, pp. 160-75, 2017.

[18] H. Shabani, B. Vahidi, and M. Ebrahimpour, "A Robust PID Controller Based on Imperialist Competitive Algorithm for Load-Frequency Control of Power Systems", ISA Transactions, vol. 52, pp. 88-95, 2013.

[19] P. N. Topno, and S. Chanana, "Load Frequency Control of a Two-Area Multi-Source Power System using a Tilt Integral Derivative Controller", Journal of Vibration and Control, vol. 24 , pp. $110-25,2018$.
[20] K. S. Simhadri, B. Mohanty, and SK. Panda, "Comparative Performance Analysis of 2DOF State Feedback Controller for Automatic Generation Control using Whale Optimization Algorithm", Optimal Control Applications and Methods, vol. 40, pp. 24-42, 2019.

21] B. Khokhar, S. Dahiya, and KPS. Parmar, "A Novel Fractional Order Proportional Integral Derivative Plus Second-Order Derivative Controller for Load Frequency Control", International Journal of Sustainable Energy, 2020, https://doi. org/10. 1080/14786451. 2020. 1803861 .

[22] R. K. Sahu, S. Panda, and U. K. Rout, "DE Optimized Parallel 2DOF PID Controller for Load Frequency Control of Power System with Governor Dead-Band Nonlinearity", International Journal of Electrical Power \& Energy Systems, vol. 49, pp. 19-33, 2013.

23] U. K. Rout, R. K. Sahu, and S. Panda, "Design and Analysis of Differential Evolution Algorithm Based Automatic Generation Control for Interconnected Power System", Ain Shams Engineering Journal, vol. 4, no. 3, pp. 409-421, 2013.

[24] BVS. Acharyulu, B. Mohanty, and P. K. Hota, "Analysis of Moth Flame Optimization Optimized Cascade Proportional-IntegralProportional-Derivative Controller with Filter for Automatic Generation Control System Incorporating Solar Thermal Power Plant", Optimal Control Applications and Methods, pp. 1-16, 2020.

[25] D. Guha, PK. Roy, and S. Banerjee, "Symbiotic Organism Search Algorithm Applied to Load Frequency Control of MultiArea Power System", Energy Systems, vol. 9, pp. 439-68, 2018.

[26] R. K. Sahu, TS. Gorripotu, and S. Panda, "Automatic Generation Control of Multi-Area Power Systems with Diverse Energy Sources using Teaching Learning-Based Optimization Algorithm", Engineering Science and Technology, an International Journal, vol. 19, pp. 113-34, 2016.

27] TS. Gorripotu, DV. Kumar, MK. Boddepalli, and R. Pilla, "Design and Analysis of BFOA Optimized PID Controller with Derivative Filter for Frequency Regulation in Distributed Generation System", International Journal of Automation and Control, vol. 12, no. 2, pp. 291-323, 2018.

[28] S. Padhan, R. K. Sahu, S. Panda, "Application of Firefly Algorithm for Load Frequency Control of Multi-Area Interconnected Power System", Electric Power Components and Systems, vol. 42, pp. 1419-30, 2014

[29] R. Storn, "Differrential Evolution - a Simple and Efficient Adaptive Scheme for Global Optimization over Continuous Spaces", Technical report, International Computer Science Institute, 11, 1995.

Received 4 September 2020

Jagan Mohana Rao Chintu is currently working towards the PhD degree at the Department of Electrical Engineering, VSSUT, Burla, Sambalpur, Odisha, India. His research interests include application of soft computing techniques in power system Engineering.

Rabindra Kumar Sahu received the $\mathrm{PhD}$ degree from the Indian Institute of Technology Madras, Chennai, India. He is presently working as a Professor in EEE Deptt., VSSUT, Burla, Odisha, India. His research interests comprise soft computing techniques to Electrical Engg. application.

Sidhartha Panda received PhD degree from IIT, Roorkee, India. He is currently working as a Professor in EE Deptt., VSSUT, Burla, Odisha, India. His areas of research include Soft computing, FACTS, Power System Stability. 